\title{
DIE DRUSENMINERALIEN
}


Verlag von VEIT \& COMP. in Leipzig.

\section{EDELSTEIN KUNDE.}

Bestimmung und Unterscheidung der Edelsteine und Schmucksteine. Die künstliche Darstellung der Edelsteine.

Von

Dr. C. Doelter,

o. ö. Professor der Mineralogie an der k. k. Universität Graz.

Mit zahlreichen Abbildungen im Text.

gr. 8. 1893. geh. 5 H.

\section{ERDBEBENKUNDE.}

Die Erscheinungen und Ursachen der Erdbeben.

Die Methoden ihrer Beobachtung.

Von

Dr. Rudolf Hoernes,

o. ö. Professor der Geologie und Palaeontologie an der Universität Graz.

Mit zahlreichen Abbildungen und Karten im Text nebst zwel Tafeln.

gr. 8. 1893. geh. 10 h.

BEITRÄGE ZUR KENNTNIS

DES

VERRUCANO.

Von

Dr. L. Milch,

Privatdozenten der Mineralogie an der Universität Breslau.

2 Teile.

Mit einer Tabelle.

gr. 8. 1892 u. 1896 . geh. 8 A6.

\section{LITHIA HERCYNICA.}

Verzeichnis

der

Minerale des Harzes und seines Vorlandes.

Von

Dr. phil. Erwin Schulze.

8. 1895. geh. 4 * 20 F. 


\section{DIE \\ DRUSENMINERALIEN \\ DES \\ STRIEGAUER GRANITS.}

VoN

DR. AR'THUR SOHWANTKE.

LEIPZIG,

VERLAG VON VEIT \& COMP.

1896. 
Druck von Metzger \& Wittig in Leipzlg. 